\title{
Desde el compromiso de las transferencias
}

\author{
Josep María Bonet Bertomeu
}

Arbor CLXXX, 710 (Febrero 2005), 417-429 pp.

\section{Introducción}

En Enero de 2002, los Servicios Sanitarios gestionados por el INSALUD fueron transferidos a las diez Comunidades Autónomas que todavía no los habían recibido. Se completó así el mapa territorial de Servicios de Salud Autonómicos, si exceptuamos Ceuta y Melilla que siguen gestionados por la Administración Central.

Habiendo transcurrido más de tres años desde aquella fecha se puede ver con cierta perspectiva aquel proceso, algunos de sus componentes y aportar así algo al momento que vive nuestro sistema, siempre con ánimo de enriquecer el debate.

Las reflexiones en este sentido se pueden considerar oportunas por coincidir en un momento singular, en el que nuestro sistema tiene en su agenda importantes retos para decidir su futuro: la financiación, el papel del ciudadano y del profesional en la configuración del sistema, los futuros escenarios,...

Trataré, en consecuencia de recordar alguno de los antecedentes del proceso, para posteriormente referirme al futuro inmediato e intentar así contribuir en algo en la elaboración de propuestas para su abordaje.

En la construcción del llamado Estado de las Autonomías, aparece de forma recurrente quien cuestiona en mayor o menor grado las decisiones que permiten avanzar hacia un mayor grado de descentralización de competencias, como es el caso de las transferencias sanitarias. Para explicar bien el proceso transferencial y la disolución del INSALUD es necesario un análisis preliminar de los orígenes del sistema sanitario. Las necesarias referencias las encontramos en nuestro marco constitucional, 
pues explican bien la configuración actual . La Constitución Española, en su art 43 reconoce el derecho de protección a la salud y encomienda a los poderes políticos organizar y tutelar la salud pública a través de las medidas preventivas y de las prestaciones y servicios necesarios. El sistema, como característica definitoria, se asienta sobre la configuración del modelo de aseguramiento único que de un modelo de Seguridad Social evoluciona posteriormente hacia un modelo de Sistema Nacional de Salud financiado en su mayor proporción a través de los presupuestos generales.

Es en el título VIII del Texto Constitucional donde se define la ordenación territorial del Estado en la que se posibilita la asunción de competencias en materia de sanidad. El Estado se reserva la regulación de las bases y la coordinación general de la sanidad.

Estas previsiones constitucionales fueron desarrolladas por la Ley General de Sanidad, Ley 14/86, estableciéndose los principios básicos que configuran el Sistema Nacional de Salud:

- Carácter público, universal, y gratuito

- Derechos y deberes de los ciudadanos y de los poderes públicos

- La descentralización política de la sanidad

- La integración de las diferentes estructuras y servicios públicos al servicio de la salud en el SNS

- La organización en áreas de salud

- Desarrollo del Modelo de Atención Primaria

Se puede interpretar en consecuencia, que la configuración actual del Sistema Sanitario español es el resultado de esta integración de la totalidad de los Servicios de Salud autonómicos transferidos, fruto de un proceso de descentralización política de la sanidad que en conjunto ha llevado más de 20 años.

\section{El Proceso Transferencial}

Las transferencias de las competencias en la gestión de los servicios de salud tienen características diferentes según pertenezcan a la primera etapa (1981-1994), o al proceso más reciente 2002. Las primeras a su vez fueron diferentes en cada caso. Entre la primera, Cataluña, y la última Canarias transcurrieron quince años, se realizaron transferencias a siete Comunidades Autónomas siendo diferentes las correlaciones de fuerzas políticas prácticamente en todas ellas. 


\section{Desde el compromiso de las transferencias}

Las transferencias se acompañaron además de las respectivas negociaciones financieras entre la Administración Central del Estado y la Autonómica. Como hoy sabemos, salvo en el caso de las Comunidades Forales, los resultados de la negociación no aportaron la estabilidad y sostenibilidad que habría cabido esperar en procesos de negociación bilaterales.

En las transferencias de 2002, los procesos se iniciaron con la modificación en 1998 de los Estatutos de Autonomía en cada una de las diez Comunidades Autónomas con servicios hasta ese momento gestionados por el INSALUD, haciéndose así formalmente competentes a partir de ese momento para recibir las transferencias. Se expresó así la clara voluntad de planificar y gestionar sus propios servicios en cada territorio autonómico independientemente del partido gobernante en cada caso.

En el año 2001 finalizaba la vigencia de la fórmula de financiación de la Sanidad, hasta entonces de carácter finalista y estable en cada escenario plurianual. Es conocida una cierta controversia acerca de la idoneidad de seguir con la financiación finalista de los Servicios de Salud aunque mejorando sus componentes y características de la formulación, o bien ir a la fórmula de financiación general de la Administración Autonómica contemplada en la LOFCA. La valoración de una y otra debería llevar a identificar aquella que además de mejorar el déficit presupuestario diera más estabilidad al sistema, generara menos incertidumbres para el siguiente período, y fuera en definitiva más aceptable para cada uno de los ámbitos territoriales. La coincidencia a fin de 2001 entre el fin de la vigencia de la fórmula para la financiación de la sanidad con el de la vigencia de la financiación de las Comunidades Autónomas fue decisiva para optar por el nuevo modelo, hoy vigente, que incluye la financiación de la sanidad en la financiación general de las comunidades autónomas. Sin duda, esta decisión se vio reforzada con el escenario pretransferencial de aquel momento, pues podía por primera vez diseñarse un escenario que englobara al total de Servicios de Salud en la financiación autonómica sin vincularlos con servicios de la Administración General del Estado, en escenarios estables de corresponsabilidad de las diferentes administraciones. Cabe añadir que una vez desaparecido el INSALUD la formulación finalista perdía parte importante de su sentido.

Así, a diferencia de los procesos anteriores, las transferencias se hicieron en bloque y tras haberse aprobado con meses de antelación, en el Consejo de Política Fiscal y Financiera, la financiación de las Comunidades Autónomas con la sanidad incorporada, por lo que la negociación bilateral en materia sanitaria quedó de hecho desvirtuada. 
En su última etapa el INSALUD había continuado con la estrategia de años precedentes de la nivelación de servicios, es decir, en la senda de disminuir las diferencias entre los territorios de gestión directa en términos de gasto corriente y de inversión per cápita. Esta fue desde su creación en 1978 una de las constantes en la gestión del Instituto. Ello fue posible gracias a gestionar la información territorializada y sometida a un constante benchmarking, de información estructural y de producción. Como se sabe, tanto el propio INSALUD gestión directa como los Servicios Autonómicos de Salud, utilizaron la información para poder comparar sus avances y también sus dificultades.

Los grandes paradigmas que definieron el modelo de atención hace ya 20 años permitieron el desarrollo de unas estrategias de atención a la población y la consolidación de una red de servicios que en conjunto constituyen ese patrimonio único en relación a otros sistemas sanitarios de nuestro entorno inmediato.

La asunción de competencias por parte de las autonomías no debería ser impedimento para reconocer en el INSALUD elementos que configuraron un organismo gestor que aún hoy podría ser referente para el conjunto del sistema, entre los cuales se podría destacar:

- Eficiencia, a través de instrumentos como los contratos programas, contratos de gestión para la asignación de recursos, control del gasto, contención del déficit,..

- Definiciones de cartera de servicios en base a necesidad y equilibrio territorial

- Bajos coste de transacción en sus competencias como Servicios Centrales de un territorio extenso

- Avance importante en la separación de funciones entre el ámbito político y el gestor, con profesionalización de la gestión y contención de la «interferencia» política en los ámbitos de gestión de los centros, hospitales y atención primaria, logrando un equipo de gestores bien cohesionado

- Profundización a lo largo de los años en la adecuación de perfiles a los puestos y en la formación continuada contribuyendo a elevar un alto grado de conocimiento experto en el sector

- Desarrollo y consolidación de Sistemas de Información, que han permitido su utilización como referente incluso hasta este momento (CMBD INSALUD 2001) a falta de nuevos referentes globales

Nuestro Sistema Sanitario sigue siendo tres años después de haber "sido transferidos la totalidad de los servicios, no sólo un muy eficaz sis- 


\section{Desde el compromiso de las transferencias}

tema de atención a las necesidades de salud de los ciudadanos sino uno de los principales mecanismos de cohesión social, y de redistribución de riqueza del país.

Si hoy alguien planteara la existencia de amenazas sobre nuestro sistema, estas lo serían sobre lo que efectivamente podemos considerar como patrimonio para todos los ciudadanos de este país, independientemente de su origen, lugar de trabajo o residencia.

La retórica que se observa con cierta frecuencia como defensa del sistema, podría desenfocar la atención sobre otras dimensiones de la problemática, o transmitir mensajes equívocos, que actuarán a la larga como amenazas más sutiles pero igualmente perniciosas para el sistema. El ciudadano y los profesionales deberían poder relacionarse con la administración en base a una relación madura, superando viejos paternalismos ilustrados. De nuevo, como vemos con acontecimientos recientes, las necesidades de los ciudadanos deberían ser las que marcaran la evolución del sistema, y no agendas, si no ocultas sí poco transparentes, de dudoso valor en la oportunidad, adecuación o aceptabilidad para el ciudadano desvirtuando así el valor de la democracia representativa.

En este sentido, creo necesario reformular los principios de avance del sistema en base a la responsabilidad del ciudadano, y de los profesionales, que deberían disponer de mejores espacios de encuentro con los ámbitos de la Política (Policy) y administración de servicios.

La capacidad de aportar elementos de impulso a los componentes del sistema de atención a la salud, parte necesariamente de un adecuado análisis del momento, en el espacio y en el tiempo, con todos los actores: ciudadanos, profesionales, gestores y políticos. Estos últimos con la responsabilidad que tienen ante los ciudadanos de nuestro país que priorizan en los primeros lugares a la Sanidad dentro de lo que debería ser la responsabilidad política.

No se trata aquí, ni sería yo el más indicado, para hablar de las deficiencias del proceso transferencial, de las consecuencias sobre el conjunto del Sistema Nacional de Salud, o de lo que se podría haber hecho y no se hizo, o de lo que se hizo y no debiera haberse hecho. Sería este, a mi modo de ver, un ejercicio estéril.

La transferencia ha logrado acercar las decisiones al ciudadano, y mejorar por tanto la relación entre administradores, ciudadanos y profesionales.

Mencionaré tan solo alguno de los elementos que, bien adquirieron cierta relevancia en el último año del INSALUD, aunque su influencia en el proceso de transferencias fuera tangencial. 
Los nuevos modelos de gestión: Se denominaron así a las nuevas formas jurídicas bajo las cuales se organizaba la gestión de los nuevos hospitales públicos. En Cataluña existían ya formulaciones diferentes al marco común de gestión administrativa y de los Estatutos de Seguridad Social en el resto del Estado, pero estaban en relación al propio origen de los hospitales, que los diferenciaba de aquellos de Seguridad Social. En Andalucía se habían desarrollado servicios bajo la fórmula jurídica de empresa pública que aportaba elementos innovadores frente a las fórmulas tradicionales de gestión en el marco administrativo. En Galicia y más tarde en el INSALUD se desarrollaron Fundaciones para la gestión de nuevos hospitales. En la Comunidad Valenciana, es conocida la fórmula de Concesión para la explotación de hospitales en régimen privado por sociedades, en las que participa una aseguradora privada, para la atención a una población determinada.

Años atrás (1991) el Informe «Abril» para la modernización del Sistema Nacional de Salud ya recomendaba la incorporación de nuevas fórmulas y nuevos instrumentos para la gestión de los servicios de salud. Pese a que tan solo se aplicaron estas formulaciones a los hospitales de nueva creación, la experiencia, a falta de una evaluación objetiva, podría considerarse como aceptable. Eso sí, independientemente de la formula jurídica utilizada. Si esta fuera la hipótesis, podríamos aventurar los elementos que los ciudadanos aprecian en la mejora de los servicios y que relación tiene ésta con la formulación jurídica, con el instrumento, que lo hace posible. Lo mismo podríamos aplicar en lo referente a los profesionales. Llamaba la atención entonces como se contraponían las opiniones y valoraciones acerca de los diferentes modelos dependiendo de la opción política y su opción de modelo. Pese a las diferencias de marco jurídico existentes, se podría decir que cualquiera de los dos principales modelos contrapuestos -la empresa pública y la fundacióneran igualmente válidos y similares para los fines de un hospital público, pudiéndose así mismo observar similares déficit en uno y en otro. Habiendo sido gestor de ambos modelos, recalcaría sus similitudes, pero especialmente su principal virtud como es el aportar nuevos instrumentos, nuevas fórmulas organizativas, valores, flexibilidad, que en conjunto faciliten un mejor entorno de trabajo para los profesionales y redunde en una mejor atención, más eficaz, más digna, y confortable. Todo ello condicionó en cierta medida el desarrollo de nuevas fórmulas de gestión en el último año del INSALUD. Los dos nuevos hospitales que entraron en funcionamiento en ese periodo lo hicieron bajo la fórmula de fundación (Ley 30), ya que no afectaba a personal previamente vinculado al Sistema. 
No obstante, la asignatura pendiente se mantuvo en la aplicación de los instrumentos de transformación de los hospitales tradicionales del INSALUD. El Decreto 29/2000 que posibilitaba esta transformación en las llamadas equívocamente Fundaciones Públicas, no se llegó a aplicar en este sentido. En un escenario transferencial un proceso de cambio de estas características no podía o no debía ser iniciado por la administración que, aunque sin duda legitima para hacerlo, iba a transferir los servicios en el mejor de los casos al final del proceso si no a medio camino en la transformación, con las repercusiones que podía comportar para el personal del centro, patronato e instrumentos de gestión.

El proceso de consolidación del personal interino, que como se sabe fue regulado por Ley, fue generado por una circunstancia excepcional, como es el no poder satisfacer en catorce años el derecho a la adjudicación de plazas estatutarias a una importante proporción de profesionales de los hospitales pese a venirlas ocupando con carácter interino. La normativa vigente o su utilización no fue por tanto un instrumento adecuado al fin de generar estabilidad en el empleo. El volumen y calidad del problema, en un escenario pretransferencial se convirtió en una exigencia al INSALUD de solución del problema. Alguien podría pensar que la consolidación estatutaria fue inadecuada frente a la "otra gran opción» como habría sido la laboralización. Sería muy largo el relacionar los motivos por los que no se optó por esta solución, pues fueron amplios y diversos, pero mencionaré el que quizás pueda entenderse mejor. La laboralización, si se pudiera plantear como la fórmula más adecuada para el Sistema debería plantearse como una solución estratégica, explícita, y bien evaluada, no como instrumento para solucionar un problema en el marco estatutario.

\section{Retos del futuro}

La oferta de servicios no ha dejado de aumentar, en términos de oferta instalada más accesible a la población (mayor número de hospitales y centros de salud,..), incorporación de tecnología en electromedicina o farmacia,... y efectivamente podemos reconocer entre las cualidades de nuestro Sistema de Salud:

- la inmediatez de la Atención

- la capacidad resolutiva

- la creciente capacidad de absorción de nuevas demandas

- la innovación tecnológica

- el papel del sistema en la redistribución de riqueza 
- favorecedor de la cohesión social

Todo ello debería preservarse en cualquiera de los escenarios previsibles de futuro, para lo cual deberá abordarse adecuadamente uno de los mayores retos, la Sostenibilidad del sistema sanitario

\section{Mejora de la Financiación y Mejora de la Eficiencia}

En la actualidad, la mejora del Sistema de Financiación se ha convertido en el eje central del debate político en el ámbito del salud. Parecería en ocasiones que con una mejora en la financiación quedan resueltos los problemas del sistema. En paralelo podrían pasar por alto componentes del modelo de salud, de gran calado en términos de salud o de bienestar social, y con evidente repercusión en los componentes del gasto. Las dificultades financieras, aunque importantes y objetivas, no deberían tampoco ocultar ineficiencias del sistema que deberíamos corregir. Deberían mantenerse los niveles de exigencia en la gestión de servicios y la evaluación de sus resultados con la implantación de instrumentos de gestión, para lo cual debería mantenerse los niveles de exigencia y adecuación de perfiles profesionales a los gestores del sistema.

En la evolución reciente de nuestro sistema sanitario el problema de la suficiencia financiera ha estado siempre presente aunque con grados de intensidad variables. De hecho con la vigencia de la anterior fórmula de financiación del INSALUD y de los Servicios Autonómicos de Salud transferidos excepto los de régimen foral, se podía observar como al inicio de cada período, y tras la correspondiente negociación, la aportación financiera suponía en efecto una inyección de recursos adicionales que permitían financiar programas nuevos en el período, pero también financiar servicios ya consolidados. La pretendida suficiencia financiera al inicio era seguida en años sucesivos de crecimientos del gasto a un ritmo superior al PIB saturando la capacidad financiera y superándola al final del período con la correspondiente generación de déficit.

Pero quizás conviene recordar que incluso en los años 80, cuando todavía no estaban vigentes fórmulas estables de financiación del INSALUD, la incorporación de instrumentos de gestión (Unidades de medida de la producción intermedia, Contratos de gestión, Codificación de los procedimientos,..) en nuestros hospitales o una planificación de recursos muy rigurosa en Atención Primaria de Salud, permitieron sostener el sistema de salud aún en escenarios muy desfavorables. Debemos añadir, por su importancia en aquel momento pero también porqué marcó con 


\section{Desde el compromiso de las transferencias}

claridad la gestión corporativa, los esfuerzos de los ámbitos profesionales en incorporar los conceptos e instrumentos de la gestión clínica, con la interiorización de los conceptos de coste por parte de los profesionales de la salud, la selección adecuada de gestores de servicios de salud y la formación de estos en conceptos de gestión de servicios públicos. Son todos ellos claros ejemplos de cómo la mejora de la financiación debe ir acompañada de medidas corporativas en los servicios de salud encaminadas a la mejora de la eficiencia.

La transferencia de los servicios de salud a todas las Comunidades Autónomas, excepto Ceuta y Melilla, pudo presuponer:

- En unos casos, la voluntad de contar con todos los servicios especializados y disponer de toda la tecnología para poder desarrollar todos los procedimientos diagnósticos y terapéuticos. Esto es, sin necesidad de derivar a su población a ninguna otra Comunidad Autónoma

- En otros, singularmente Madrid y Cataluña, la necesidad de que se financiaran los servicios hospitalarios de referencia, hasta ese momento de nivel nacional, que podían ser utilizados por ciudadanos de otras comunidades autónomas y en consecuencia alguien debía sufragar el gasto incurrido

Efectivamente, el nuevo escenario, que traía consigo un nuevo marco de relaciones entre Comunidades Autónomas y entre estas y la Administración Central del Estado, dio lugar a la creación del fondo de cohesión social y a la propia Ley 16 / 2003 de Cohesión y Calidad del Sistema Nacional de Salud. A pesar de haber logrado tácitamente una identificación entre todos los 17 servicios de salud y evitar así su impermeabilización para los ciudadanos, tanto por su cuantía como por los criterios de reparto parecería oportuno replantear su formulación, en el marco actual del debate sobre financiación de la sanidad.

En el mismo sentido, los mecanismos de financiación de la atención a ciudadanos comunitarios (residenciados, transeúntes, turistas,..) merecería mejor atención ya que los prestadores de servicios, aquellos que incurren en el gasto, son las Comunidades Autónomas, aunque afecta a todas ellas de forma muy desigual. El receptor de los fondos es la Administración del Estado (Ministerio de trabajo/INSS). En consecuencia los incentivos para una correcta facturación por parte de los servicios dónde se realiza la prestación o para las administración autonómica, son inexistentes. Las características de nuestro sistema, y la facilidad de acceso a los servicios de Hospitales y de Atención Primaria para los ex- 
tranjeros, incluso para recibir prescripciones farmacéuticas, podría estar generando perversiones muy costosas a algunas Comunidades Autónomas sin que se esté cuantificando la compensación financiera o la corrección en su caso de los gastos soportados.

Todo ello debería ir en consonancia con los necesarios ajustes posttransferenciales de las Consejerías o Departamentos de salud, Servicios Autonómicos y Organizaciones territoriales. Sería oportuno realizar un Plan de Servicios bien para el conjunto del territorio autonómico bien para áreas concretas que requieren de reformulaciones por cambios demográficos importantes o por presiones externas al propio ámbito de los servicios de salud de titularidad pública, evitando así incrementalismo y crecimientos desordenados insostenibles.

Se deberían reformular las estrategias de nuestros centros sanitarios, a ser posible tras la evaluación de los planes estratégicos que con toda seguridad se realizaron en los últimos años. Nos encontraremos en muchos casos en los que realmente no ha habido un seguimento del grado de consecución de las estrategias formuladas o de los resultados relacionados con estas. Por esta razón la nueva definición, implantación y gestión de las estrategia debería enlazarse con objetivos, iniciativas e indicadores que permitan su seguimiento y el conocimiento a cerca del grado de consecución de resultados.

La evolución de los Sistemas públicos de prestación de servicios de salud tiene como meta la atención a la salud de los ciudadanos en su sentido más amplio. Las medidas que se han comentado serán necesarias para la mejora de la eficiencia lo deben ser sin menos cabo de la calidad de la prestación.

La mejora de la calidad debería empezar por hacer accesibles a los ciudadanos información de cada centro sobre los resultados en las principales prestaciones sanitarias y su propia gestión. Con ello se lograría aportar más información y sobre el funcionamiento de los servicios sanitarios.

En este sentido es compatible con la actual tendencia de desarrollo empresarial hacia la sostenibilidad o desarrollo de la cultura corporativa en las empresas excelentes: La eficiencia, la necesidad de incorporar a las ciudadanos en las decisiones de las políticas sanitarias, una política social adecuada y comprometida

\section{La red de conocimiento y la red de servicios}

En los últimos años se ha interiorizado en nuestro país, y es en consecuencia una realidad, la necesidad desarrollar redes de conocimien- 
to en el campo de la investigación. Sería recomendable avanzar en la configuración del Sistema en Red, con la riqueza que ello supone intrínsecamente, para permitir compartir conocimiento y favorecer el desarrollo profesional frente al clásico desarrollo de los servicios con estructuración de carácter piramidal.

La información a cerca de la actividad asistencial o de la gestión de servicios, o en relación a las características y modalidades de atención y los instrumentos utilizados son materias que se mantienen en compartimentos estancos, celosamente guardados, en sus respectivos ámbitos. Sin duda el benhmarking ha ayudado a avanzar en los servicios de salud razón por la cual sería deseable avanzar en el debate a cerca de los servicios, sus modalidades y sus instrumentos con el ánimo de generar mecanismos para compartir la información que puede ayudar a mejorar aprendiendo de lo que otros ya han desarrollado. Deberíamos potenciar el actual Instituto de Información Sanitaria bajo un enfoque hacia la información compartida a cerca de servicios, instrumentos y resultados que a la vez facilite el acceso de esta información a los ciudadanos. Sería deseable que esto nos permitiera la publicación sistemática en Internet de los datos útiles para su uso por los servicios de salud y sus diferentes niveles asistenciales, con un cierto grado de estandarización para permitir la comparabilidad y la usabilidad. Permitiría mejorar los niveles actuales de debate sobre el futuro de nuestro sistema de salud y adecuar los desarrollos tecnológicos (TIC) de forma armónica.

\section{Ciudadanos, Profesionales y Administración}

Deberíamos contribuir, como se ha mencionado con anterioridad, a identificar mejor los espacios de corresponsabilidad y encuentro entre los ciudadanos, los profesionales y la administración para mejorar el debate social en todos sus componentes: La sostenibilidad del sistema, el acceso a prestaciones, la redistribución, equidad, cohesión social,

Los niveles alcanzados por nuestras organizaciones sanitarias, la incorporación tecnológica, el conocimiento médico acreditado, los resultados en términos de salud, y la valoración que de todo ello tienen los ciudadanos, reclaman un nuevo impulso en términos organizativos que adecue la organización a los profesionales y los dote de mejores instrumentos para realizar mejor su función.

Hemos visto como en ocasiones déficit organizacionales o de modelo, son suplidos con iniciativas de profesionales que estando al nivel de las circunstancias dan una respuesta adecuada a las necesidades de los ciu- 
dadanos. Podríamos pensar que es necesario mejorar el marco de relación y el equilibrio existente entre ciudadanos, administración y profesionales. Cuando se potencia una de las líneas relacionales en detrimento de las otras, por oportunismo, demagogia o desconexión de la realidad de los ciudadanos según el caso, el sistema pierde su equilibrio y afecta a todos sus componentes y sin duda a los resultados.

o Desafección en los responsables políticos

o Efectos acomodaticios de los gestores

o Desincentivación profesional

o Escepticismo en los ciudadanos

La necesaria identificación de los servicios a prestar y su interrelación, independientemente de la titularidad de los servicios requerirá una adecuada Organización de ámbito territorial. El modelo organizativo debe ser el adecuado para transmitir las políticas en atención a la salud, en investigación, en desarrollos tecnológicos, gestión del conocimiento, desarrollo profesional, y en los instrumentos y modalidades de gestión. Los centros sanitarios deberían dotarse de los instrumentos de gestión adecuados, independientemente de su titularidad, que les permita mejorar su autonomía de gestión y optimización de resultados . Esto implica un cierto grado de descentralización en busca de niveles más efectivos en la gestión sin que ello implique necesariamente cambios en su personalidad jurídica.

No obstante si parece inaplazable la

- identificación de órganos de gobierno con presencia territorial para cada centro, que permita la definición adecuada de las normas de funcionamiento interno,

- las relaciones estables con el financiador,

- la definición de estrategias

- garantía de sostenibilidad (financiera, social, ambiental)

- rendición pública de cuentas y

- resultados en términos de salud, a disposición de los ciudadanos

todo ello de acuerdo a la necesidad y al nivel y disponibilidad del conocimiento y las tecnología médicas

Sin falsos temores podríamos encontrar la solución si-fuéramos conscientes del estado actual del sistema, de su diagnóstico. De lo contrario 


\section{Desde el compromiso de las transferencias}

podría hacerse de la ausencia virtud, la omisión, la inacción puede ser en ocasiones quien sustituya, por inexistente, a la política (Policy).

Hoy tenemos a nuestra disposición potentes instrumentos en sistemas de información que en manos de nuestros profesionales permitirán

o una gestión clínica efectiva con optimización de recursos y que posibilite la evaluación de los recursos empleados

- utilización de guías de práctica clínica

- acceso a los ítems del paciente desde cualquier punto

- nuevas tecnologías que permitan nuevos espacios relacionales entre el ciudadano y el médico / organización de salud

En relación a los gestores del sistema creo que se hace cada vez más necesario un recambio generacional manteniendo la exigencia de los elevados perfiles profesionales que han venido siendo la característica fundamental en los años de desarrollo de nuestro sistema, y que no debería verse comprometida por la cercanía, que en el escenario transferido, existe entre políticos y gestores a nivel local. Para reforzar lo anterior, debería retomarse la senda de la formación de directivos en habilidades gestoras, en negociación, innovación, dirección de equipos,.,..

Por último, la defensa de nuestro Sistema Nacional de Salud debería llevarnos a la búsqueda de nuevas formulaciones, su debate y la necesaria evaluación rigurosa. Con la distancia podemos ver hoy multitud de elementos que han hecho evolucionar al sistema en una buena dirección y con parámetros de gasto asumibles para nuestro nivel de renta. Debemos pues seguir avanzando en la sostenibilidad, con instrumentos innovadores, que permitan a los profesionales dar a los ciudadanos la atención sanitaria que se merecen y exigen. 